\title{
Do Wolf-Rayet stars have similar locations in hosts as type lb/c supernovae and long gamma-ray bursts? ${ }^{\star}$
}

\author{
G. Leloudas ${ }^{1}$, J. Sollerman ${ }^{1,2}$, A. J. Levan ${ }^{3}$, J. P. U. Fynbo ${ }^{1}$, D. Malesani ${ }^{1}$, and J. R. Maund ${ }^{1,4,5}$ \\ 1 Dark Cosmology Centre, Niels Bohr Institute, University of Copenhagen, Juliane Maries Vej 30, 2100 Copenhagen $\varnothing$, Denmark \\ e-mail: giorgos@dark-cosmology.dk \\ 2 The Oskar Klein Centre, Department of Astronomy, Stockholm University, AlbaNova, 10691 Stockholm, Sweden \\ 3 Department of Physics, University of Warwick, Coventry CV4 7AL, UK \\ 4 Department of Astronomy \& Astrophysics, University of California, Santa Cruz, CA 95064, USA \\ 5 Sophie \& Tycho Brahe Fellow
}

Received 27 November 2009 / Accepted 31 March 2010

\section{ABSTRACT}

\begin{abstract}
Aims. We study the distribution of Wolf-Rayet (WR) stars and their subtypes with respect to their host galaxy light distribution. We thus want to investigate whether WR stars are potential progenitors of stripped-envelope core-collapse supernovae (SNe) and/or long-duration gamma-ray bursts (LGRBs).

Methods. We derived the relative surface brightness (fractional flux) at the locations of WR stars and compared with similar results for LGRBs and SNe. We examined two nearby galaxies, M 83 and NGC 1313, for which a comprehensive study of the WR population exists. These two galaxies contain a sufficiently large number of WR stars and sample different metallicities. To enable the comparison, the images of the galaxies were processed to make them appear as they would look at a higher redshift. The robustness of our results against several sources of uncertainty was investigated with the aid of Monte Carlo simulations.

Results. We find that the WC star distribution favours brighter pixels than the WN star population. WC stars are more likely drawn from the same distribution as SNe Ic than from other SN distributions, while WN stars show a higher degree of association with $\mathrm{SNe} \mathrm{Ib}$. It can also not be excluded that WR (especially WC) stars are related to LGRBs. Some differences between the two galaxies do exist, especially in the subtype distributions, and may stem from differences in metallicity.

Conclusions. Although a conclusive answer is not possible, the expectation that WR stars are the progenitors of SNe Ib/c and LGRBs survives this test. The trend observed between the distributions of WN and WC stars, as compared to those of SNe Ib and Ic, is consistent with the theoretical picture that SNe Ic result from progenitors that have been stripped of a larger part of their envelope.
\end{abstract}

Key words. supernovae: general - stars: Wolf-Rayet - gamma-ray burst: general

\section{Introduction}

To understand the origin of cosmic explosions like supernovae ( $\mathrm{SNe}$ ) and gamma-ray bursts (GRBs), it is important to study and constrain their environments. Fruchter et al. (2006; hereafter F06) presents a sample of 32 long-duration GRB (LGRB) host galaxies and has developed a new technique to show that LGRBs have a tendency to occur in the brightest pixels of their host galaxies. This contrasts to a comparison sample of core-collapse (CC) $\mathrm{SNe}$ where the $\mathrm{SN}$ locations instead follow the light distribution of their hosts. Kelly et al. (2008; hereafter K08) further shows that not all CC SNe follow the same host galaxy light distribution, with SNe Ic strongly skewed towards the brightest regions of their hosts. The SN Ic population is thus broadly consistent with that of LGRBs. Indeed, SNe Ic, and in particular broad-lined SNe Ic, are so far the only type that have been been firmly observationally connected to LGRBs (Galama et al. 1998; Hjorth et al. 2003; Stanek et al. 2003; Malesani et al. 2004) or their lower energy siblings, X-ray flashes (Pian et al. 2006; Campana et al. 2006; Sollerman et al. 2006).

Using a similar method, Anderson \& James (2008) mapped the association of nearby $\mathrm{SNe}$ with $\mathrm{H} \alpha$ regions. They find that

* Table A.1 is only available in electronic form at http: //wWw . aanda.org
SNe Ic trace the $\mathrm{H} \alpha$ emission, and recent star formation, to a higher degree than other CC SN types. This was attributed to $\mathrm{SNe}$ Ic having more massive progenitors than $\mathrm{SNe} \mathrm{Ib}$, which are in turn more massive than SNe II. Larsson et al. (2007) modeled the F06 results and derived minimum masses for $\mathrm{CC} \mathrm{SNe}$ and LGRBs of 8 and $20 M_{\odot}$, respectively. A theoretical-modeling approach was also taken by Raskin et al. (2008), who uses the F06 method and a simulated solar-metallicity spiral galaxy to predict the (increasing) minimum cut-off masses of the progenitors of $\mathrm{SNe}$ II and Ic.

The next step is to expand this type of study to the potential progenitors of these cosmic explosions. The most plausible candidates are Wolf-Rayet (WR) stars (Woosley \& Bloom 2006; Crowther 2007). In single-star evolutionary models, WR stars are the final phases in the life of very massive stars (minimum initial mass $>22-37 M_{\odot}$, depending on the metallicity and rotation; Meynet \& Maeder 2005), which have shed their hydrogen envelope. WR stars can also result from close binary star interaction, through Roche-lobe overflow, in which case the minimum mass can be decreased down to $\sim 15 M_{\odot}$ (Eldridge et al. 2008). A comprehensive review of WR stars is given by Crowther (2007). Here we restrict ourselves to giving the background information that is essential for the purposes of this study: WR stars are further divided into nitrogen-rich (WN) and carbon-rich (WC) stars. WN and WC stars are believed to 
give rise to supernova explosions of type $\mathrm{Ib}$ and Ic, respectively (Crowther 2007; Georgy et al. 2009). Depending on their emission line properties, width, and appearance, they can be further divided into "early" or "late" types (WNE, WNL, WCE, and WCL, respectively). The WR star populations strongly depends on metallicity, with both the total number of WR stars and the relative ratio of WC/WN stars increasing dramatically with metallicity (Crowther 2007; Meynet \& Maeder 2005). This is attributed to the dependence of winds on metallicity, which leads to much more effective mass loss in the presence of metals (Vink $\&$ de Koter 2005).

Our purpose here is to study the distribution of WR stars and their subtypes within their hosts, using the method applied by F06 and K08. The main motivation is the following: if WR stars are the immediate progenitors of $\mathrm{SNe} \mathrm{Ib} / \mathrm{c}$ and LGRBs, then we would also expect their distributions with respect to the host galaxy light to be similar. Since the progenitors of $\mathrm{SNe} \mathrm{Ib/c} \mathrm{still}$ evade direct detection (see e.g. Maund et al. 2005; Crockett et al. 2008; Smartt 2009), this method can give us hints to their nature. The same is true for LGRBs, for which a direct progenitor detection seems impossible, at least in the near future. It is noteworthy that WR features have been clearly identified in the host of the most nearby LGRB (GRB 980425/SN 1998bw), albeit with a considerable offset to the explosion position (Hammer et al. 2006; Christensen et al. 2008) and more recently in four more LGRB hosts (Han et al. 2010).

This paper is structured as follows. In Sect. 2 we discuss the galaxies chosen for studying the WR distribution. In Sect. 3 we outline the methods used throughout the paper, and in Sect. 4 we present our results. Section 5 contains a discussion of the results and several uncertainty factors, and Sect. 6 summarizes our conclusions.

\section{Galaxy selection}

It is essential for our purposes to use galaxies where the WR star population has been mapped accurately and systematically in an unbiased way, as much as that is possible. For this reason we used the results of Crowther and collaborators, who have recently identified and spectroscopically classified the WR stars in a number of galaxies beyond the Local Group, in an essentially complete way (Schild et al. 2003; Hadfield et al. 2005; Hadfield \& Crowther 2007). The method used is explained in detail in, e.g., Hadfield \& Crowther (2007) and includes the following steps: first imaging of the galaxy is obtained and then regions with candidate WR stars are identified by their excess in a narrow-band He II $\lambda 4684$ filter over the continuum. Subsequently, the regions containing WR stars are confirmed with spectroscopy of the candidates. Their spectral type (WN, WC, early, late) is determined, and the number of WR stars is estimated by fitting template spectra to the flux-calibrated, integrated spectrum of the WR-star region. By excluding galaxies where the survey did not cover the entire galaxy (Schild et al. 2003; Hadfield \& Crowther 2006), we decided to rely on the following two galaxies: M 83 (Hadfield et al. 2005) and NGC 1313 (Hadfield \& Crowther 2007).

We did not use the existing catalogues for other (very nearby) galaxies, such as the LMC (Breysacher et al. 1999), the SMC (Massey et al. 2003), or other Local Group galaxies (Massey \& Johnson 1998), because they suffer from several incompleteness-related issues (chance discoveries, Malmquist bias) and have complicated revision histories (see e.g. Massey \& Johnson 1998). Another practical disadvantage with these galaxies is their large angular size. The WR galaxy catalogue in Schaerer et al. (1999) is also unsuitable for our purposes, because it is a list of diverse objects that have been defined from the appearance of a broad He II feature in their integrated spectra.

The number of WR stars contained in the two selected galaxies $(\sim 1000$ and 100 , respectively) exceeds the number of 32 LGRB used by F06 and $44 \mathrm{SNe}$ Ib/c used by K08 (out of 504 SNe of all types). While F06 and K08 constructed their distributions by looking at one explosion site per galaxy for many galaxies, discovered in searches that are not unbiased themselves, we look at many (potential) explosion sites in a few galaxies. Such a comparison should be valid, as long as the galaxies we choose are not different from the typical galaxies studied by K08 and F06. While this statement is obviously more problematic for the high-redshift F06 hosts (see also discussion in K08), we can compare the global properties of M 83 and NGC 1313 (see Sects. 2.1 and 2.2) to those of the K08 SNe Ib/c host sample. M 83 turns out to be a galaxy very typical of the K08 sample in terms of absolute magnitude, metallicity (Fig. 1), and morphological type - with its only difference that it is closer to us. NGC 1313 is somewhat different, although such galaxies are present in the K08 sample: fainter, metal-poor, and more irregular, it is more reminiscent of the LGRB hosts of F06. It is important for our study, as we will see, that the two galaxies probe two different metallicity limits.

Below, a more detailed description of the individual galaxies used in this study is given, containing only those details necessary for our discussion. Some of their key properties are also summarized in Table 1.

\section{1. $M 83$}

M83 is a nearby $(4.5 \pm 0.3 \mathrm{Mpc})$, Milky-Way type $\mathrm{SAB}(\mathrm{s}) \mathrm{c}$ spiral galaxy with a super-solar metallicity ${ }^{1}$ of 9.0 (Hadfield et al. 2005, and references therein). In total, 132 WR regions containing $\sim 1000 \pm 300 \mathrm{WR}$ stars were spectroscopically confirmed by Hadfield et al. (2005), while 89 more regions are labeled as candidates, still awaiting spectroscopic follow-up. As part of the same study, Crowther et al. (2004) points out the large number of WCL stars found in M 83, directly related to its high metallicity.

Hadfield et al. (2005) were not able to look for WR stars in the nucleus of M 83 (owing to saturation in the images). However, K08 also provide results after the removal of the bulge, for galaxies with a significant bulge contribution, so comparison is still possible. For our analysis we used a wide field image obtained using the ESO/MPI $2.2 \mathrm{~m}$ (+WFI).

While very different from the LGRB host galaxies (F06), M 83 is a prodigious SN producer, with 6 observed $\mathrm{SNe}$ in the 20th century. Although most of them remain unclassified, one of them is a prototypical SN Ib (SN 1983N; Uomoto \& Kirshner 1985; Elias et al. 1985). The host site of SN $1983 \mathrm{~N}$ is not associated with a confirmed WR site and is $\sim 7 "$ away from the nearest candidate region. If an isolated WR star (such as several others identified in M 83) had been responsible for the 1983 explosion, it would of course have disappeared (e.g. Maund \& Smartt 2009) from the frames obtained later by Hadfield et al. (2005).

\subsection{NGC 1313}

NGC 1313 is a SB(s)d spiral at a distance of $4.1 \pm 0.1 \mathrm{Mpc}$ and has a metallicity of 8.23, i.e., intermediate between the SMC and the LMC (Hadfield \& Crowther 2007, and references therein).

\footnotetext{
1 Metallicities in this paper are expressed as oxygen abundances $\log (\mathrm{O} / \mathrm{H})+12$.
} 
Table 1. Key properties of M 83 and NGC $1313^{a}$.

\begin{tabular}{|c|c|c|c|c|c|c|c|c|c|c|}
\hline Galaxy & $\begin{array}{c}\text { Metallicity } \\
(\log (\mathrm{O} / \mathrm{H})+12)\end{array}$ & $\begin{array}{c}D \\
(\mathrm{Mpc})\end{array}$ & $\begin{array}{l}\text { Pix. scale } \\
\left(\mathrm{pc}^{2} \mathrm{pix}^{-1}\right)\end{array}$ & $\begin{array}{c}M_{B} \\
(\mathrm{mag})\end{array}$ & $\begin{array}{c}M_{K} \\
(\mathrm{mag})\end{array}$ & Morph. & Conf. reg. ${ }^{c}$ & $\begin{array}{c}\mathrm{WN}^{d} \\
\mathrm{WNE}+\mathrm{WNL}\end{array}$ & $\begin{array}{c}\mathrm{WC}^{d} \\
\mathrm{WCE}+\mathrm{WCL}\end{array}$ & Cand. reg. ${ }^{e}$ \\
\hline M 83 & 9.0 & $\frac{1}{4.5}$ & $5.3^{2}$ & -20.2 & -23.7 & $\mathrm{SAB}(\mathrm{s}) \mathrm{c}$ & 132 & $232+250$ & $28+526$ & 89 \\
\hline NGC 1313 & 8.23 & 4.1 & $4.0^{2}$ & -18.9 & -20.1 & $\mathrm{SB}(\mathrm{s}) \mathrm{d}$ & 70 & $29+22$ & $34+0$ & $12^{f}$ \\
\hline
\end{tabular}

Notes. ${ }^{(a)}$ Compiled from Hadfield et al. (2005); Hadfield \& Crowther (2007) and NED. ${ }^{(b)}$ In the images from ESO/MPI 2.2 m (+WFI) and VLT/FORS used in the analysis. A galaxy at $z=0.01$ in the SDSS $2.5 \mathrm{~m}$ images used by K08 would have $\sim 80^{2} \mathrm{pc}^{2} \mathrm{pix}^{-1}$. ${ }^{(c)}$ Confirmed regions containing one or more WR stars. ${ }^{(d)}$ Total number of stars per subtype used in this paper. These numbers may differ slightly from those reported in the literature, for the following reasons: WN5-6 stars have been included in the WNE distribution; WO in WCE; transitional WN/WC have been included in both WN and WC. The total number of WR stars is obtained by adding their corresponding subtypes. ${ }^{(e)}$ Candidate regions for which spectroscopy has not been conducted. ${ }^{(f)} 11$ of these are photometrically consistent with WN stars.
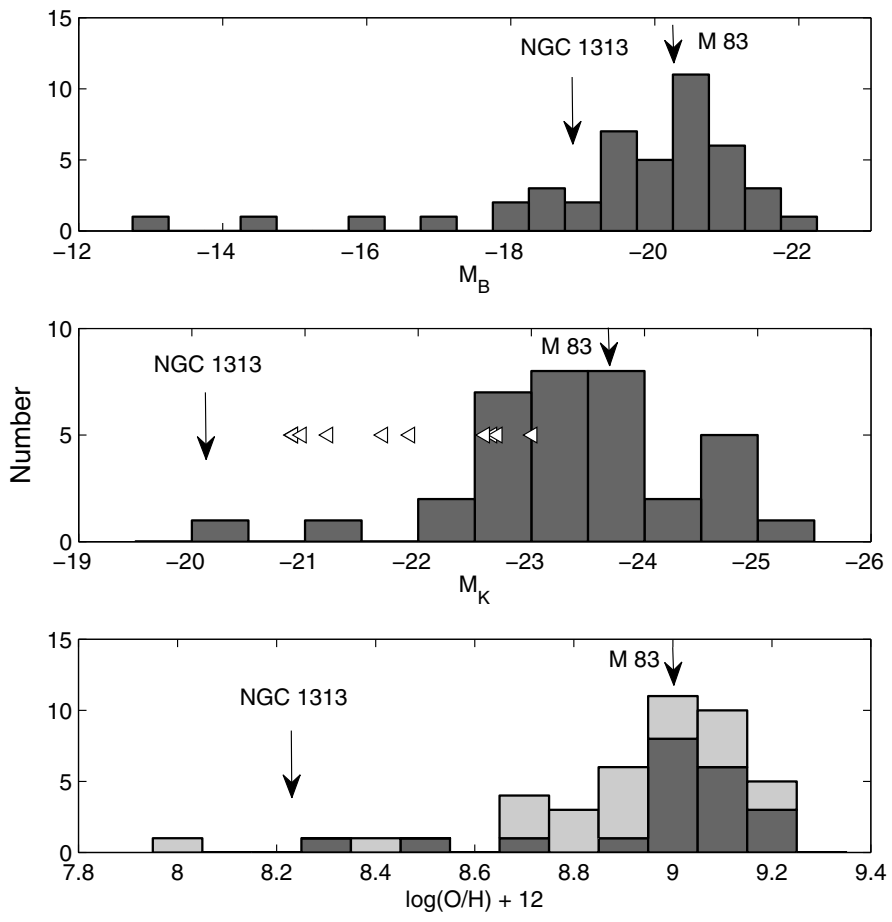

Fig. 1. Global properties of M 83 and NGC 1313 compared to those of the $\mathrm{K} 08 \mathrm{SNe} \mathrm{Ib} / \mathrm{c}$ hosts. Histograms of the absolute magnitudes $M_{B}, M_{K}$ (a better tracer of the total stellar mass), and metallicity are presented. The information presented here was collected from NED, SDSS, and from Prieto et al. (2008), for the metallicities. These are global metallicities on the scale used by Tremonti et al. (2004). The $M_{K}$ histogram is incomplete towards the faintest galaxies and upper limits, compatible with the 2MASS survey upper limit, are provided (white arrows). To get an indicative value for the galaxies with no metallicity reported in Prieto et al. (2008), i.e. the ones not included in SDSS DR4, we used an approximate luminosity-metallicity relation (see Sect. 3.4). These galaxies are represented by the light-grey shaded histogram. The corresponding values for M 83 are marked, indicating that this galaxy is very typical of the K08 sample, just closer. NGC 1313, on the other hand, is a galaxy that is fainter and more metal-poor, hence more reminiscent of LGRB hosts.

This galaxy is more reminiscent of the LGRB hosts than M 83, both regarding the more irregular shapes of the LGRB hosts (F06) and their, usually, low metallicity (Sollerman et al. 2005; Modjaz et al. 2008; Savaglio et al. 2009). Two type II SNe have been recorded in this galaxy, SNe 1962M and 1978K. Hadfield \& Crowther (2007) report on the spectroscopic confirmation of 70 WR regions (success rate of $85 \%$ over the candidates followed with spectroscopy), while 12 more regions remain candidates and are photometrically consistent with WN stars. Unlike M 83, few of the identified regions contain more than one WR star, with their total number estimated to be between 84 and 115 .

Our analysis was performed on the VLT/FORS images obtained by Hadfield \& Crowther (2007). We included the single WO star (star \#31) $)^{2}$ in the WC stars, while the transitional WN/C star \#11 was included in both the WN and WC distributions. For the WN5-6 stars we adopted a WNE classification, although this choice is not unique (Crowther 2007). The results presented in this paper do not change, however, if we include them in the WNL distribution instead.

\section{Methods}

\subsection{Fractional flux}

As fractional flux of a pixel belonging to a galaxy, we define the sum of all counts in pixels less bright than the pixel in question, over the sum of all counts in all the pixels belonging to the galaxy. This is the same definition as the one used by F06 and K08.

\subsection{Pixel detection}

As in F06 and K08, the SExtractor software (Bertin \& Arnouts 1996) was used to identify the pixels that belong to the galaxies. The parameters used were similar to the ones in these references to make the comparison as close as possible. We used $B$-band images, which correspond to the same (rest-frame) wavelength window examined by F06 and K08.

\subsection{Pre-processing of images}

Identification of individual stars in other galaxies requires that the galaxies are nearby. As a consequence, the apparent dimensions of M 83 and NGC 1313 (at $z=0.0017$ and 0.0016 , respectively) are much larger than the SN hosts of K08, and their images cover thousands of pixels on the CCD. While usually an advantage, for our type of analysis this can pose problems in two different ways. First, a considerable number of foreground stars are superimposed on the image and contribute to the pixel fractional fluxes. It is desirable to remove the foreground star pointspread functions (PSF), but it is not straightforward to identify which stars are in the foreground and which belong to the host galaxy. It has been shown that it is possible to distinguish between massive stars in other galaxies and foreground dwarfs by use of colour-colour diagrams (Massey 1998; Massey et al. 2009) or other kinematical techniques involving spectroscopy

\footnotetext{
${ }^{2}$ We follow the numbering of Hadfield et al. (2005) and Hadfield \& Crowther (2007).
} 
(Drout et al. 2009). However, it was not necessary to resort to such detailed techniques for our purposes, because it is sufficient to remove only the brightest stars that are clearly in the foreground and contribute with significant light. To this end, all stars brighter than 20 mag were removed from our images by subtracting their PSF with tasks in the package daophot in $\mathrm{IRAF}^{3}$. At the distances of our galaxies, there is no degeneracy below this limit, and all stars can safely be considered Galactic. To assess the effect of stars fainter than $20 \mathrm{mag}$, we performed a Monte Carlo (MC) simulation: the expected number of foreground stars in the field (Bahcall \& Soneira 1981) and their corresponding counts, per magnitude bin, were removed randomly and repetitively from our images. This experiment showed that our results are not sensitive to their presence.

Second, each of our pixels contains light from a much smaller physical area than the ones studied by K08: the pixel scale in our images is $\sim 4$ and 5 pc (along a pixel side) for NGC 1313 and M 83, respectively. On the other hand, the $\mathrm{SN} \mathrm{Ib/c} \mathrm{hosts} \mathrm{of} \mathrm{K08} \mathrm{have} \mathrm{a} \mathrm{median} \mathrm{redshift} \mathrm{of} z \gtrsim 0.01$, which corresponds to a distance of $\sim 42 \mathrm{Mpc}$ for $H_{0}=$ $72 \mathrm{~km} \mathrm{~s}^{-1} \mathrm{Mpc}^{-1}$. At this distance, one pixel on the SDSS $2.5 \mathrm{~m}$ telescope, used by K08, corresponds to an area of $\sim 80 \times 80 \mathrm{pc}^{2}$. Consequently the pixel fractional fluxes they quote refer to these large areas. To enable the comparison we thus binned our images by factors of 15-19 in order to "bring our galaxies" to a redshift of $z=0.01$. Subsequently, the images were convolved with a Gaussian in order to simulate the typical seeing of 3.2 pixels measured on the K08 SDSS plates. The final images are shown in Fig. 2.

\subsection{The metallicity dependence}

As mentioned above, both the total number of WR stars and the WC/WN ratio increase drastically with metallicity. This trend is also observed for M 83 and NGC 1313 (Hadfield et al. 2005; Hadfield \& Crowther 2007). If WR stars are the progenitors of $\mathrm{SNe} \mathrm{Ib} / \mathrm{c}$, we therefore expect to observe relatively more $\mathrm{SNe} \mathrm{Ib} / \mathrm{c}$ in galaxies with high than low metallicity. This has, in fact, been shown by Prantzos \& Boissier (2003), Prieto et al. (2008) and Boissier \& Prantzos (2009). Anderson \& James (2009) even claim that they can disentangle the metallicity dependence from the mass dependence and that $\mathrm{SNe}$ Ic come from higher metallicity environments than $\mathrm{SNe} \mathrm{Ib}$. On the other hand, LGRBs are often found in low-metallicity environments (Sollerman et al. 2005; Stanek et al. 2006; Savaglio et al. 2009; Levesque et al. 2010a), especially in comparison to broad-lined SNe Ic that are not associated with known LGRBs (Modjaz et al. 2008). However, it has not been completely settled whether all LGRB hosts are metal poor or if we are simply missing more metal-rich hosts owing to a dust-obscuration bias (e.g. Fynbo et al. 2009; Levesque et al. 2010b; Svensson et al. 2010). Because of these strong metallicity dependencies, in the following section, our results will be presented separately for the metalrich M 83 and the metal-poor NGC 1313.

We have also attempted to look for possible metallicity signatures in the K08 sample. To do so, the K08 SN type Ib and Ic fractional flux distributions were divided into two equal number bins of "high" and "low" metallicity. For the host galaxies for which Prieto et al. (2008) do not report metallicities, we followed Prantzos \& Boissier (2003) in using the galaxy global luminosity as a proxy for metallicity. Although it has been shown

${ }^{3}$ IRAF is distributed by the National Optical Astronomy Observatory: http://iraf.noao.edu/iraf/web/

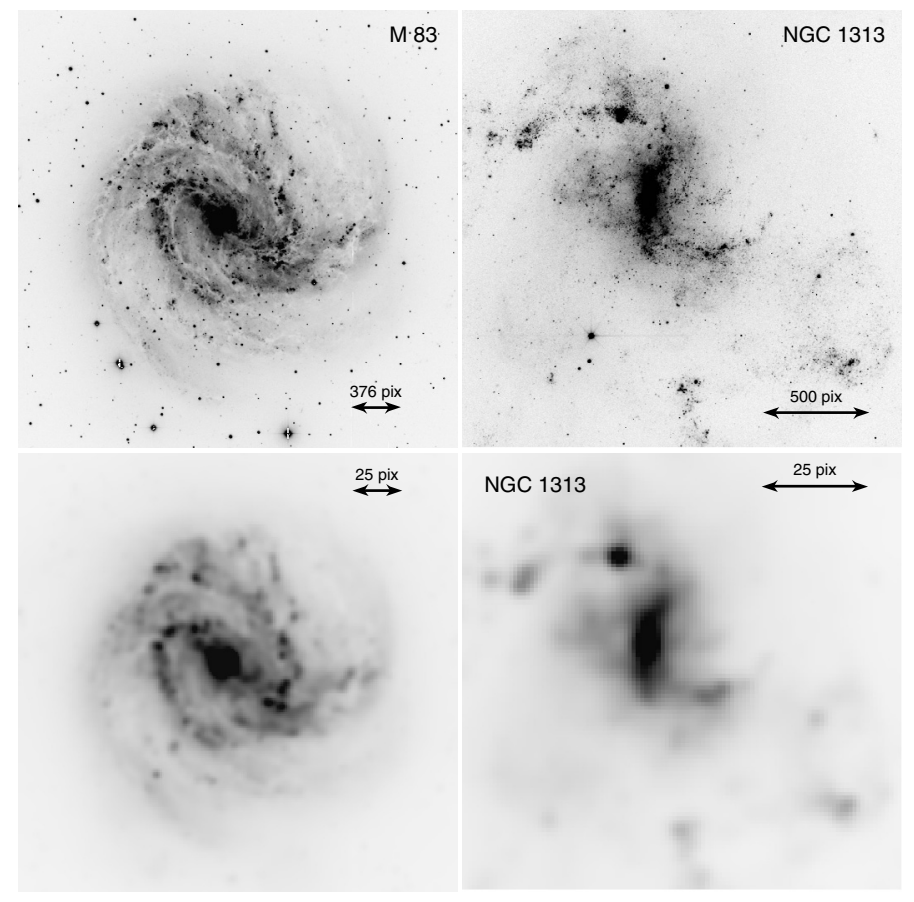

Fig. 2. Top left: ESO/MPI $2.2 \mathrm{~m}$ (+WFI) $B$-band image of M $83(z=$ $0.0017, D=4.5 \mathrm{Mpc})$. The displayed part is $3300 \times 3300$ pixels. Top right: VLT/FORS $B$-band image of NGC $1313(z=0.0016$, $D=4.1 \mathrm{Mpc}$ ). The image is $2048 \times 2048$ pixels. Bottom: processed images, which simulate how the galaxies above would look at $z=0.01$ at the SDSS $2.5 \mathrm{~m}$ telescope. Bright foreground stars have been removed and the images have been binned such that one pixel side is $\sim 80 \mathrm{pc}$ along. A Gaussian filter has been used to smooth the resulting images to an $F W H M$ of $\sim 3.2$ pixels, typical of the SDSS frames. The pixel dimensions of the resulting images are $220 \times 220$ and $108 \times 108$, respectively. In each panel, a double arrow spanning $2 \mathrm{kpc}$ across has been drawn and the corresponding pixel scale is noted.

that the local metallicities at the explosion sites can differ from the global metallicity or its proxies (e.g. Modjaz et al. 2008; Thöne et al. 2009), this should not pose any problems for the rough separation of the sample in two bins. It was thus assumed that the galaxies without reported metallicities have metallicities consistent with the best fit luminosity-metallicity relation, as derived from the galaxies with reported metallicities (lightgrey histogram in Fig. 1, lower panel). We see no convincing evidence that the high and low metallicity SNe are different in terms of their fractional flux distributions. This investigation is, however, clearly limited by the small number of SNe in the sample and by the absence of very low (i.e. subsolar) metallicities.

\section{Results}

To compare with the fractional flux distributions of LGRBs (F06) and various types of SNe (K08), it is necessary to consider the number of WR stars contained within each WR confirmed region, because each one of them is a potential progenitor of a SN and/or LGRB explosion. Hadfield et al. (2005) and Hadfield $\&$ Crowther (2007) give estimates for the numbers or WR stars contained in each region, as well as for the corresponding errors. The number of stars per region is simply taken into account by, e.g., including the fractional flux value of the pixel that hosts 5 WR stars 5 times in the corresponding distribution.

The pixel fractional fluxes measured at the locations of the WR stars, together with their number and subtype, are listed for the two galaxies in Table 2. In Figs. 3 and 4 (left panels), we have 
Table 2. Fractional fluxes at the locations of the WR $\operatorname{stars}^{a}$.

\begin{tabular}{rrrrr}
\hline \hline & $\mathrm{ID}^{b}$ & Subtype $^{c}$ & Number & $F F^{d}$ \\
\hline \multirow{4}{*}{ M 83 } & 1 & WNL & 3 & 0.29 \\
& 2 & WCL & 2 & 0.34 \\
& 3 & WCL & 2 & 0.26 \\
& 4 & WNE & 16 & 0.48 \\
& 5 & WCL & 8 & 0.48 \\
& 6 & WCE & 3 & 0.06 \\
& $\ldots$ & $\ldots$ & $\ldots$ & $\ldots$ \\
& 133 & cand. & $\ldots$ & 0.25 \\
NGC 1313 & 134 & cand. & $\ldots$ & 0.54 \\
\hline & 1 & WNE & 1 & 0.37 \\
& 2 & WCE & 1 & 0.30 \\
& 3 & WNL & 1 & 0.06 \\
& $\ldots$ & $\ldots$ & $\ldots$ & $\ldots$ \\
& 28 & cand. & $\ldots$ & 0.38 \\
\hline
\end{tabular}

Notes. ${ }^{(a)}$ An indicative portion of this table is shown. For the full table refer to the electronic version of the Journal. ${ }^{(b)}$ We follow the exact numbering of Hadfield et al. (2005) and Hadfield \& Crowther (2007). Additional info (e.g. fluxes, errors in star numbers) can be found in these references. ${ }^{(c)}$ "cand". stands for candidate region. ${ }^{(d)}$ These fractional fluxes refer to a binning of 15 and 19 for the images of M 83 and NGC 1313 we have used, respectively. Although we have shown that the results do not change significantly with the choice of binning, individual fractional fluxes can vary.

plotted the WN and WC fractional flux distributions for the two galaxies, together with the data from F06 and K08. Table 3 contains the corresponding Kolmogorov-Smirnov (KS) test $p$-values that two distributions are drawn from the same parent distribution. To keep the graph as uncrowded as possible, we focused on the most relevant WR and SN types. The table, however, contains more information including the division of WR stars into their subtypes.

The null hypothesis of the KS test is that two samples are drawn from the same distribution and the purpose of the test is to reject (or not) this null hypothesis. The KS test should not be used to deduce new physics, but rather for the opposite purpose, namely to test whether an idea with strong theoretical background, such as that WR stars and some CC SNe are associated, can be rejected. In that respect, a definite rejection of the null hypothesis requires a $\mathrm{p}$-value $<0.3 \%$. Doubts can exist for $\mathrm{p}$-values $<5 \%$, but these are certainly not enough to disprove a well-justified hypothesis. Inspired by the Gaussian distribution, from now on we call these significance levels (or rather the very similar $0.3 \%$ and $4.6 \%$ ) the $3 \sigma$ and $2 \sigma$ levels at which the hypothesis can be rejected, although this is purely a naming convention. For higher p-values, including the (Gaussian) $1 \sigma$, or $31.7 \%$ limit, there is very weak evidence against the null hypothesis.

To better illustrate this, in Figs. 3 and 4 (right panels) we have colour-mapped the KS p-values from Table 3 reflecting their significance levels. The only conclusive result $(>3 \sigma$ exclusion) is coloured in red. Orange, yellow, and green show the progressive decreasing significance at which the hypothesis of common parent distribution can be rejected. In that context, yellow is more probable than orange, but even orange cannot be excluded by the present data. From now on, when we refer to our "results", we are mostly refering to these significance levels and their relative order.

\subsection{High metallicity $-M 83$}

For the metal-rich galaxy M 83, which is typical of the K08 SNe $\mathrm{Ib} / \mathrm{c}$ sample, we make the following observations.

- The distribution of WR stars as a whole is consistent with those of SNe Ib/c (yellow in Fig. 3) or even LGRBs (orange). It is however inconsistent with SNe II (red).

- The distribution of WC stars is more consistent with $\mathrm{SNe}$ Ic ( $p=31.5 \%$, almost green) than with any other type of SN ( $p<4.6 \%$, which occurs for SNe Ib). From the WC subtypes, it is mostly the WCL stars that are responsible for this association. The less abundant WCE stars, on the other hand, seem to better follow the host galaxy light and are more consistent with $\mathrm{SNe}$ that behave in a similar way.

- WN stars are more consistent with $\mathrm{SNe} \mathrm{Ib}$ (yellow) than with other kinds of supernovae (orange or red). We caution, however, that the early and late WN distributions behave quite differently. By excluding WNL stars, which might not be direct SN progenitors (see Sect. 5), WNE stars alone show a clearer preference to $\mathrm{SNe} \mathrm{Ib}$ and their association to $\mathrm{SNe}$ Ic can be ruled out with a certainty of over $3 \sigma$.

- SNe II and Ia, which follow their host galaxy light distribution well (K08), show no association (null hypothesis excluded at over $3 \sigma$ ) with most WR stars, with the possible exception of early subtypes that seem to occupy fainter locations than their late counterparts. The same claim, but not as strict, could be made for the high-redshift CC SN sample of F06 (which most likely consists of SNe II).

To study how the errors in the number of WR stars can affect the results above, we have followed an MC approach. Multiple realizations of the distributions were generated where the numbers of WR stars per region were drawn randomly from Gaussian distributions with the mean and standard deviation specified by the number of WR stars and the associated errors provided by Hadfield et al. (2005). While this causes the p-values to fluctuate around their central values in Table 3, none of the qualitative conclusions above are affected. By this, we mean that p-values rarely jump to another $\sigma$ significance level (i.e. their colour in Fig. 3 does not change) and that their relative ordering remains the same. The standard deviations on the p-values scale with the p-values themselves: typical fluctuations are of the order of $\pm 6 \%$ for a $\mathrm{p}$-value of $26 \%$ or $\pm 1 \%$ for a p-value of $3.4 \%$. Of course, if the number estimates of Hadfield et al. (2005) are biased in a systematic way, significant changes might be expected. The results may also be susceptible to changes in the limited number of SNe and LGRBs in the comparison samples.

The above results were obtained after removing the bulge contribution of M 83 and comparing with the corresponding results of K08. This is because we have no detailed information about the nuclear WR population of this galaxy, and a bulge can be visually identified and removed. The bulge light removal was done, similar to K08, by placing a circular ring around the bulge and by replacing all bulge pixels with the mean pixel value inside the ring.

\subsection{Low metallicity - NGC 1313}

In the case of the metal-poor NGC 1313, we observe the following:

- The global WR population is this time mostly consistent with $\mathrm{SNe} \mathrm{Ib}$ (at a highly significant p-value), while at the same time an association with $\mathrm{H}$-rich $\mathrm{SNe}$ is probable as well.

- This is especially obvious in the case of WN stars that trace well the light of the host galaxy. WN stars are again mostly 

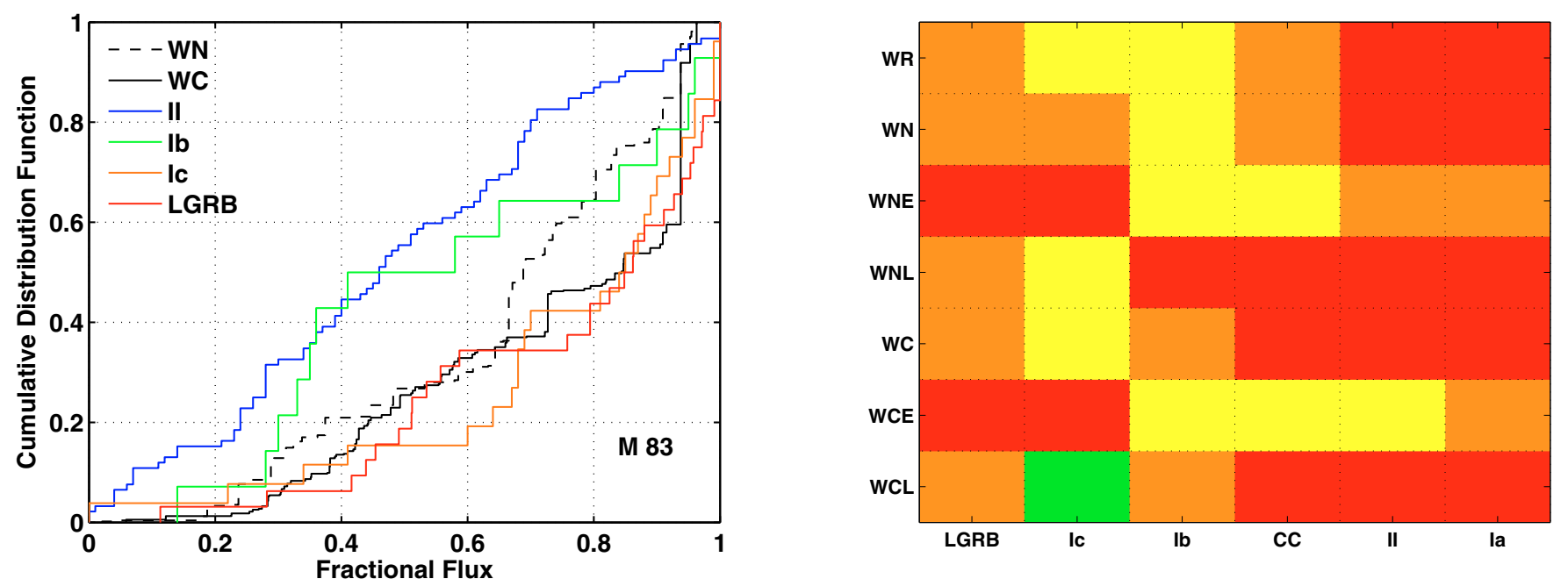

Fig. 3. Left panel: the distribution of WN and WC stars in M 83, a galaxy with properties typical of the K08 SN Ib/c sample, with respect to their location on their host light, plotted with the distributions of LGRBs and SNe II, Ib, and Ic (F06, K08). Right panel: this colour map indicates at what significance level we can exclude that a SN or LGRB explosion is associated to a certain (sub)type of a progenitor WR star. Red shows p-values $<0.3 \%$, orange $0.3<p<4.6 \%$, yellow $4.6<p<31.7 \%$, and green $p>31.7 \%$ (Table 3). The comparison has been done after the removal of the bulge, because no detailed information about the nuclear WR population exists and a bulge can be visually identified and removed (K08). The WC distribution is more skewed towards brighter pixels than the WN distribution. It is more probable that WC stars are drawn from the same population as SNe Ic than SNe Ib (or any other type of SN), and it is more probable that WN stars are associated to SNe Ib than with SNe Ic (or any other type of SN). This is consistent with theoretical predictions (Crowther 2007; Georgy et al. 2009).

consistent with $\mathrm{SNe} \mathrm{Ib}$, while a relation to $\mathrm{SNe}$ Ic can be excluded this time at high significance. On the other hand, their association to SNe II cannot be excluded any more, but instead shows a high probability. Again, differences are seen between the WNE and WNL distributions.

- WC stars, on the other hand, show significant association probabilities with SNe Ic and even LGRBs. We recall here that the WC population at the metallicity of NGC 1313 consists entirely of WCE stars.

Again, we checked on how the total WR content in the range discussed by Hadfield \& Crowther (2007) affects the distributions and we did not find any qualitative difference with the results above. In the case of NGC 1313, the numbers of WR stars per region are considerably lower than in $\mathrm{M} 83$. If region \#64 contains 6 rather than 3 WC stars, as suspected by Hadfield \& Crowther (2007), the WC distribution is pushed even closer to the $\mathrm{SNe}$ Ic and further from the SNe II (which turn red).

\section{Discussion}

From the results presented in the previous section, a qualitative pattern seems to emerge: WC stars are on average found in brighter pixels than WN stars. As a consequence, WC stars show higher probabilities of association with SNe Ic. On the other hand, WN stars are most consistent with the locations of $\mathrm{SN}$ Ib explosions. This is the main result presented in this paper and is in broad agreement with the theoretical expectations, i.e., that $\mathrm{SNe}$ Ic result from progenitors that have been stripped of a larger part of their outer envelope (Crowther 2007). Also from a statistical point of view the WN and WC distributions are almost (geographically) incompatible (at significance $>3 \sigma$ for M 83, while $p=5.9 \%$ for NGC 1313), which highlights the need to consider these two subtypes separately when discussing SN progenitors, despite their strong physical connection. Other studies, however, caution that there is no strict one-to-one correlation between progenitor and supernova type, but that leaks might exist, e.g., less massive WC stars exploding as $\mathrm{SNe} \mathrm{Ib}$ (Georgy et al. 2009).

Concerning LGRBs, they are not inconsistent with being drawn from the WR population, although typically at lower significance than $\mathrm{SNe}$ Ic. This is not a surprise since not all SNe Ic produce LGRBs (e.g. Soderberg et al. 2006). It is, however, tempting to point out that the highest p-value obtained for LGRBs (yellow) is the one for WC stars at low metallicity, in agreement with the proposed low-metallicity requirement (Yoon \& Langer 2005; Woosley \& Heger 2006).

Besides the general trend that is common for the two galaxies, differences do exist between the individual WN and WC fractional flux distributions. The difference is more pronounced in the case of the WN distributions that are almost mutually inconsistent ( $p=0.6 \%$ ). The WN distribution in NGC 1313 tracks the host galaxy light better and is more consistent with SNe II. To some degree, this difference can be attributed to the important metallicity difference between the two galaxies: Georgy et al. (2009) predict that at low metallicity (similar to NGC 1313) the highest fraction of WN stars are actually expected to explode as $\mathrm{SNe}$ II and not SNe Ib. Due to lower mass-loss, the less massive stars are still expected to leave enough hydrogen to be detectable in the explosion spectra.

Other differences, however, especially at the subtype level, are more difficult to explain. The most striking is related to the WNL populations of the two galaxies. In M 83 they are found on the brightest pixels, while in NGC 1313 they lie on the faintest ones. Indeed, the two WNL distributions are inconsistent with each other, at a significance $>3 \sigma$. The reason for this is unclear, but it should be mentioned that WNL stars are not always stripped-envelope massive stars. In many cases, they are very luminous $\mathrm{H}$-rich WN stars that are still burning $\mathrm{H}$ in their core. They are therefore in a phase preceding the LBV phase and not direct progenitors of SNe Ib/c (Crowther 2007; Smith \& Conti 2008). According to Crowther (2007), a possible way to distinguish between H-rich WNL stars and stripped WNL stars is that the former usually lie in young massive clusters. One 

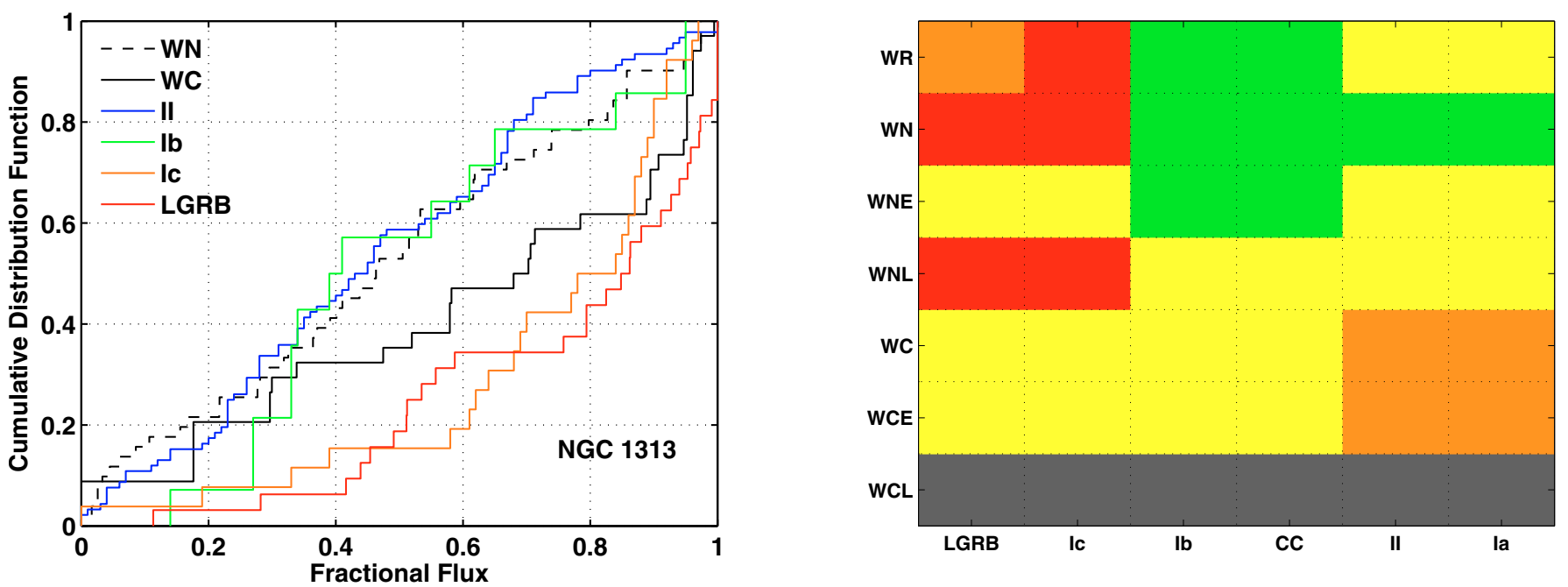

Fig. 4. The same as in Fig. 3 but for NGC 1313, a more metal-poor and irregular galaxy, probably similar to a low-redshift counterpart of the high-redshift F06 hosts. The colour-coding in the right panel is the same as in Fig. 3, but grey has been used for WCL stars that are not present in this galaxy.

Table 3. KS test p-values (\%).

\begin{tabular}{llrrrrrr}
\hline \hline & & LGRB & Ic & Ib & CC & II & Ia \\
\hline & WR & 1.2 & 20.7 & 8.3 & 2.1 & 0.0 & 0.0 \\
& WN & 0.7 & 2.4 & 16.6 & 1.8 & 0.0 & 0.0 \\
M 83 & WNE & 0.0 & 0.0 & 5.2 & 29.4 & 1.5 & 1.5 \\
(High Z) & WNL & 3.5 & 29.8 & 0.2 & 0.0 & 0.0 & 0.0 \\
& WC & 2.0 & 31.1 & 4.6 & 0.2 & 0.0 & 0.0 \\
& WCE & 0.0 & 0.0 & 8.3 & 11.5 & 5.0 & 3.7 \\
& WCL & 2.2 & 33.0 & 2.6 & 0.1 & 0.0 & 0.0 \\
\hline & WR & 0.3 & 0.2 & 77.2 & 53.0 & 7.5 & 9.4 \\
& WN & 0.1 & 0.0 & 81.3 & 96.4 & 68.1 & 89.7 \\
NGC 1313 & WNE & 5.9 & 8.1 & 47.6 & 64.4 & 6.5 & 10.3 \\
(Low Z) & WNL & 0.0 & 0.0 & 22.9 & 25.1 & 20.0 & 15.3 \\
& WC & 17.9 & 14.5 & 22.8 & 6.1 & 1.0 & 0.8 \\
& WCE & 17.9 & 14.5 & 22.8 & 6.1 & 1.0 & 0.8 \\
& WCL & $\ldots$ & $\ldots$ & $\ldots$ & $\ldots$ & $\ldots$ & $\ldots$ \\
\hline
\end{tabular}

could argue that this is mostly the case for the WNL stars in M 83, while the ones in NGC 1313 are mostly isolated. If this is true, caution should be applied when comparing the WNL distribution of M 83 to those of SNe or LGRBs. (WNE stars alone share most of the qualitative properties discussed for WN stars in this galaxy.) On the other hand, their positions in NGC 1313 are consistent with the predictions of Georgy et al. (2009): that WNL stars are expected to give SNe II, especially at low metallicity. We may thus be probing different WNL populations in the two galaxies.

Below we assess the robustness of our results, with respect to several uncertainty factors. We call a result robust as long as the significance levels in the KS test between two distributions (in the form of colours in Figs. 3 and 4) remain unchanged and retain their relative values with respect to other p-values. Indeed, with small exceptions, this is the case for most p-values. We conclude therefore that our main results are not sensitive to these uncertainties.

\subsection{The remaining candidates' locations and their implications.}

The number and nature of the remaining candidate regions in our galaxies has until now been ignored. To determine how important that is, we have followed an MC approach. By comparing the fractional flux distribution of the candidate pixels of M 83 to the corresponding distribution of the $\mathrm{WN}$ and $\mathrm{WC}$ pixel positions (i.e. without taking the number of stars per region into account this time, since we lack this kind of information for the candidate regions) we find that the candidate locations are more likely drawn from the WN $(p=60 \%)$ than the WC $(p=0.5 \%)$ pool. A possible reason could be that WC stars have stronger narrow-band excess over the continuum and might have been preferentially selected for follow-up spectroscopy. Indeed, WN stars, especially weak-lined WNE and very late WNL types, might suffer from some incompleteness (P. Crowther, priv. comm.). For NGC 1313, Hadfield \& Crowther (2007) state that the (few) remaining candidate regions are photometrically consistent with WN stars.

In our MC simulation for M 83, we attempted many realizations where we allowed $66 \%$ of the candidates (a percentage equal to the success rate of the actual spectroscopic survey) to be genuine WR regions containing a number of stars equal to the median number of stars per confirmed WR region (plus their median error). We made simulations for the two limiting cases that the candidate regions included in the actual WR distributions were all included in the WN distribution or were divided between WN and WC stars according to the observed WC/WN ratio. The latter simulation is displayed as an example in Fig. 5. In both simulations we see only small differences in the results reported in Table 3, which do not change any of our conclusions. Similarly, we found no significant differences in our main results for NGC 1313.

\subsection{The effect of temporal evolution}

A possible objection concerning the significance between the apparent difference between the WN and WC distributions is that sometimes a WN star is just on an evolutionary path towards a WC final stage, while all WC stars have already been 


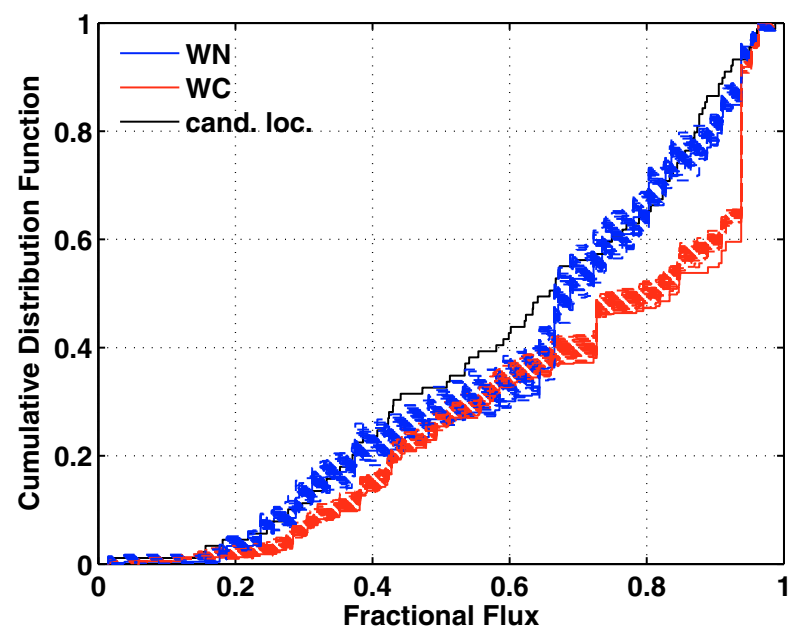

Fig. 5. Example MC simulation for the inclusion of the candidate regions in the WR distributions in M 83. Each realization results in a different $\mathrm{WN}$ and WC distribution (dashed lines), while the solid lines denote the original distributions (Fig. 3). For visual purposes, only the first 50 realizations have been plotted although the simulation contains 1000 runs. In the displayed simulation, the candidates were divided to $\mathrm{WC}$ and $\mathrm{WN}$ stars according to the observed WC/WN ratio. Our main conclusions remain unchanged, as do the colours in Fig. 3, right panel. Only the p-value between WC stars to $\mathrm{SNe}$ Ib changed colour, becoming yellow $(p=6.6 \pm 1.3 \%)$ from being (marginally) orange ( $p=4.6 \%)$. Similar MC simulations were used for both galaxies in order to assess the importance of effects like the presence of foreground stars fainter than $20 \mathrm{mag}$, that the number of stars per region have an associated error estimate, and the evolution of WN to WC stars.

through the WN phase once (Meynet \& Maeder 2005; Crowther 2007). Whether this evolution will occur at all, and the related timescale, is strongly dependent on mass and metallicity (Meynet \& Maeder 2005). The mass range for which stars actually die as WN stars is quite narrow (Georgy et al. 2009) but if convolved with the IMF their number can become important. By using the mass limits from Georgy et al. (2009; their Table 4), we estimate that, at the metallicity of NGC 1313 , only $\sim 1 / 6$ of the observed WN stars (the most massive) will evolve to WC stars. In M 83, however, we expect this ratio to increase to almost 2/3.

In an MC simulation for each galaxy, we allowed the abovementioned ratios of WN stars to be removed from the WN and be included in the WC distributions. The expected result is that the WC distribution will be pushed towards fainter pixels, since it will be contaminated by WN stars. This simulation ignores that, while some WN stars evolve to WC, some new WN stars will be born, and many stars will explode. It also ignores the related timescales (all of a few Myr), and the brightness evolution of the stars themselves and has deliberately not made any assumptions about masses based on the positional information of the stars on the galaxy. A hidden (reasonable) assumption is therefore that we do not observe these galaxies at a very special time in their existence and that, in this context, these simulations represent a limiting worst case. For both galaxies, we note that the probability of associating WC to $\mathrm{SNe}$ Ib jumps up one significance level, while their association to SNe Ic and LGRBs is reduced, although the significance remains the same. The WN central association p-values remain unchanged, as expected, while all WN and WC p-values get assigned error bars that can occasionally cross different significance levels. However, even in this limiting worst case, the relative scaling between the $\mathrm{p}$-values remains unchanged and the WC distribution is always skewed towards brighter pixels than $\mathrm{WN}$.

\subsection{How much does binning affect the results?}

To compare with results obtained for galaxies at higher redshift, our images were subject to the degradation process (binning) described in Sect. 3.3. We examine here the implications of this process.

If the fractional fluxes are measured in the original images, considerably higher values are obtained and the WR distributions become skewed towards brighter pixels, even brighter than for distant SNe Ic and LGRBs, especially in the case of WC stars. The effect of binning is that a bright isolated star (that has a high pixel fractional flux value in our original image) will be smoothed out and have a low fractional flux in the processed image. A bright association of many pixels, however, such as a cluster, will be less affected and therefore have a high fractional flux also in the processed image. This mimics the effect of distance, where isolated stars cannot be detected at higher redshift but clusters can. Although not binning would result in an apparently stronger result with regard to the probable association of WR stars to $\mathrm{SNe} \mathrm{Ib} / \mathrm{c}$ and LGRBs, this degradation is needed to make a fair comparison. In that context, if WR stars are indeed the progenitors of these explosions, the fractional flux values at the low brightness tails of the F06 and K08 distributions, are probably caused by isolated WR stars, while the ones with high fractional fluxes are those that are found in bright clusters. That these explosions tend to occur in pixels brighter than average (F06, K08) can then be explained by the preference of WR stars to be found in large associations.

We have also tested various degrees of binning to confirm that our results are not tuned to the chosen values (simulated redshift $\sim 0.01$ ). Although some small changes occur, we checked that our conclusions are robust to lower and higher values of binning as long as the PSF in the original image is not subsampled.

\section{Conclusions}

We performed a F06 type analysis on the WR populations of two nearby galaxies and compared our results to the distributions of different types of $\mathrm{SNe}$ (K08), focusing on $\mathrm{SNe} \mathrm{Ib} / \mathrm{c}$, and LGRBs (F06). M 83 is a metal-rich galaxy, typical of the K08 SNe Ib/c host sample, while NGC 1313 is more metal poor, irregular, and similar to high-redshift LGRB host galaxies. To enable the comparison we resampled our images to simulate a higher redshift.

WR stars are consistent with being the progenitors of $\mathrm{SNe} \mathrm{Ib} / \mathrm{c}$ or even LGRBs. Furthermore, the WC stars are distributed in brighter locations of their hosts than WN stars. It is therefore more likely that WC stars are the progenitors of SNe Ic and WN stars of SNe Ib, as also expected by theoretical arguments. This result is robust to a number of systematic checks that we carried out.

Although encouraging, these results are based on the only two galaxies for which such an analysis is possible at present. Even though they contain enough of WR stars and we have shown that they are, most probably, not special in any way, it would of course be desirable to validate our results on a larger galaxy sample, once such a sample becomes available. Ideally, such a sample should span a wide range of metallicities.

Acknowledgements. We thank Paul Crowther for reading the manuscript and giving us comments, as well as Jens Hjorth and Michał Michałowski for discussions. We are grateful to Davide De Martin and to the ESO ePOD department for 
G. Leloudas et al.: WR star locations in their host galaxies

providing us with a reduced image of M 83 from the ESO/MPI $2.2 \mathrm{~m}$. The Dark Cosmology Centre is funded by the Danish National Research Foundation. J.S. is a Royal Swedish Academy of Sciences Research Fellow supported by a grant from the Knut and Alice Wallenberg Foundation. J.R.M. is a Sophie \& Tycho Brahe Fellow. This research has made use of the NASA/IPAC Extragalactic Database (NED), which is operated by the Jet Propulsion Laboratory, California Institute of Technology, under contract with the National Aeronautics and Space Administration. Funding for the SDSS and SDSS-II has been provided by the Alfred P. Sloan Foundation, the Participating Institutions, the National Science Foundation, the U.S. Department of Energy, the National Aeronautics and Space Administration, the Japanese Monbukagakusho, the Max Planck Society, and the Higher Education Funding Council for England.

\section{References}

Anderson, J. P., \& James, P. A. 2008, MNRAS, 390, 1527

Anderson, J. P., \& James, P. A. 2009, MNRAS, 399, 559

Bahcall, J. N., \& Soneira, R. M. 1981, ApJS, 47, 357

Bertin, E., \& Arnouts, S. 1996, A\&AS, 117, 393

Boissier, S., \& Prantzos, N. 2009, A\&A, 503, 137

Breysacher, J., Azzopardi, M., \& Testor, G. 1999, A\&AS, 137, 117

Campana, S., Mangano, V., Blustin, A. J., et al. 2006, Nature, 442, 1008

Christensen, L., Vreeswijk, P. M., Sollerman, J., et al. 2008, A\&A, 490, 45

Crockett, R. M., Maund, J. R., Smartt, S. J., et al. 2008, ApJ, 672, L99

Crowther, P. A. 2007, ARA\&A, 45, 177

Crowther, P. A., Hadfield, L. J., Schild, H., \& Schmutz, W. 2004, A\&A, 419, L17

Drout, M. R., Massey, P., Meynet, G., Tokarz, S., \& Caldwell, N. 2009, ApJ, 703,441

Eldridge, J. J., Izzard, R. G., \& Tout, C. A. 2008, MNRAS, 384, 1109

Elias, J. H., Matthews, K., Neugebauer, G., \& Persson, S. E. 1985, ApJ, 296, 379

Fruchter, A. S., Levan, A. J., Strolger, L., et al. 2006, Nature, 441, 463

Fynbo, J. P. U., Jakobsson, P., Prochaska, J. X., et al. 2009, ApJS, 185, 526

Galama, T. J., Vreeswijk, P. M., van Paradijs, J., et al. 1998, Nature, 395, 670

Georgy, C., Meynet, G., Walder, R., Folini, D., \& Maeder, A. 2009, A\&A, 502, 611

Hadfield, L. J., \& Crowther, P. A. 2006, MNRAS, 368, 1822

Hadfield, L. J., \& Crowther, P. A. 2007, MNRAS, 381, 418

Hadfield, L. J., Crowther, P. A., Schild, H., \& Schmutz, W. 2005, A\&A, 439, 265

Hammer, F., Flores, H., Schaerer, D., et al. 2006, A\&A, 454, 103
Han, X. H., Hammer, F., Liang, Y. C., et al. 2010, A\&A, 514, A24 Hjorth, J., Sollerman, J., Møller, P., et al. 2003, Nature, 423, 847 Kelly, P. L., Kirshner, R. P., \& Pahre, M. 2008, ApJ, 687, 1201

Larsson, J., Levan, A. J., Davies, M. B., \& Fruchter, A. S. 2007, MNRAS, 376, 1285

Levesque, E. M., Berger, E., Kewley, L. J., \& Bagley, M. M. 2010a, AJ, 139, 694

Levesque, E. M., Kewley, L. J., Graham, J. F., \& Fruchter, A. S. 2010b, ApJ, 712, L26

Malesani, D., Tagliaferri, G., Chincarini, G., et al. 2004, ApJ, 609, L5

Massey, P. 1998, ApJ, 501, 153

Massey, P., \& Johnson, O. 1998, ApJ, 505, 793

Massey, P., Olsen, K. A. G., \& Parker, J. W. 2003, PASP, 115, 1265

Massey, P., Silva, D. R., Levesque, E. M., et al. 2009, ApJ, 703, 420

Maund, J. R., \& Smartt, S. J. 2009, Science, 324, 486

Maund, J. R., Smartt, S. J., \& Schweizer, F. 2005, ApJ, 630, L33

Meynet, G., \& Maeder, A. 2005, A\&A, 429, 581

Modjaz, M., Kewley, L., Kirshner, R. P., et al. 2008, AJ, 135, 1136

Pian, E., Mazzali, P. A., Masetti, N., et al. 2006, Nature, 442, 1011

Prantzos, N., \& Boissier, S. 2003, A\&A, 406, 259

Prieto, J. L., Stanek, K. Z., \& Beacom, J. F. 2008, ApJ, 673, 999

Raskin, C., Scannapieco, E., Rhoads, J., \& Della Valle, M. 2008, ApJ, 689, 358

Savaglio, S., Glazebrook, K., \& LeBorgne, D. 2009, ApJ, 691, 182

Schaerer, D., Contini, T., \& Pindao, M. 1999, A\&AS, 136, 35

Schild, H., Crowther, P. A., Abbott, J. B., \& Schmutz, W. 2003, A\&A, 397, 859

Smartt, S. J. 2009, ARA\&A, 47, 63

Smith, N., \& Conti, P. S. 2008, ApJ, 679, 1467

Soderberg, A. M., Nakar, E., Berger, E., \& Kulkarni, S. R. 2006, ApJ, 638, 930

Sollerman, J., Jaunsen, A. O., Fynbo, J. P. U., et al. 2006, A\&A, 454, 503

Sollerman, J., Östlin, G., Fynbo, J. P. U., et al. 2005, New Astron., 11, 103

Stanek, K. Z., Matheson, T., Garnavich, P. M., et al. 2003, ApJ, 591, L17

Stanek, K. Z., Gnedin, O. Y., Beacom, J. F., et al. 2006, Acta Astron., 56, 333

Svensson, K. M., Levan, A. J., Tanvir, N. R., Fruchter, A. S., \& Strolger, L. 2010, MNRAS, 405, 57

Thöne, C. C., Michałowski, M. J., Leloudas, G., et al. 2009, ApJ, 698, 1307

Tremonti, C. A., Heckman, T. M., Kauffmann, G., et al. 2004, ApJ, 613, 898

Uomoto, A., \& Kirshner, R. P. 1985, A\&A, 149, L7

Vink, J. S., \& de Koter, A. 2005, A\&A, 442, 587

Woosley, S. E., \& Bloom, J. S. 2006, ARA\&A, 44, 507

Woosley, S. E., \& Heger, A. 2006, ApJ, 637, 914

Yoon, S., \& Langer, N. 2005, A\&A, 443, 643

Pages 10 to 15 are available in the electronic edition of the journal at http://www . aanda.org 


\section{Appendix A: Online table}

Table A.1. Fractional fluxes at the locations of the WR stars. Full version of Table 2 for the online article.

\begin{tabular}{|c|c|c|c|c|}
\hline & ID & Subtype & Number & $F F$ \\
\hline \multirow[t]{57}{*}{ M 83} & 1 & WNL & 3 & 0.29 \\
\hline & 2 & WCL & 2 & 0.34 \\
\hline & 3 & WCL & 2 & 0.26 \\
\hline & 4 & WNE & 16 & 0.48 \\
\hline & 5 & WCL & 8 & 0.48 \\
\hline & 6 & WCE & 3 & 0.06 \\
\hline & 7 & WNL & 5 & 0.61 \\
\hline & 8 & WCL & 1 & 0.40 \\
\hline & 9 & WCE & 1 & 0.58 \\
\hline & 10 & WCL & 2 & 0.54 \\
\hline & 11 & WNE & 4 & 0.26 \\
\hline & 12 & WNE & 2 & 0.36 \\
\hline & 13 & WCE & 3 & 0.56 \\
\hline & 14 & WCL & 4 & 0.12 \\
\hline & 15 & WCE & 1 & 0.38 \\
\hline & 16 & WCE & 2 & 0.44 \\
\hline & 17 & WCL & 6 & 0.42 \\
\hline & 18 & WCL & 6 & 0.38 \\
\hline & 19 & WCL & 1 & 0.38 \\
\hline & 20 & WCL & 2 & 0.35 \\
\hline & 21 & WCL & 1 & 0.30 \\
\hline & 22 & WNE & 4 & 0.34 \\
\hline & 23 & WCE & 1 & 0.29 \\
\hline & 24 & WCL & 4 & 0.28 \\
\hline & 25 & WNL & 4 & 0.32 \\
\hline & 26 & WNL & 2 & 0.72 \\
\hline & 27 & WCL & 6 & 0.85 \\
\hline & 28 & WCL & 8 & 0.85 \\
\hline & 29 & WCL & 1 & 0.55 \\
\hline & 30 & WCL & 1 & 0.23 \\
\hline & 31 & WCL & 42 & 0.73 \\
\hline & 32 & WCL & 12 & 0.43 \\
\hline & 33 & WNL & 1 & 0.71 \\
\hline & 34 & WCL & 3 & 0.51 \\
\hline & 35 & WNE & 14 & 0.72 \\
\hline & 36 & WCL & 5 & 0.72 \\
\hline & 37 & WCL & 4 & 0.91 \\
\hline & 38 & WNL & 7 & 0.95 \\
\hline & 38 & WCL & 21 & 0.95 \\
\hline & 39 & WCE & 2 & 0.81 \\
\hline & 40 & WCL & 6 & 0.89 \\
\hline & 41 & WNL & 14 & 0.91 \\
\hline & 41 & WCL & 13 & 0.91 \\
\hline & 42 & WNL & 5 & 0.95 \\
\hline & 43 & WNL & 3 & 0.86 \\
\hline & 44 & WNL & 5 & 0.74 \\
\hline & 45 & WNL & 1 & 0.63 \\
\hline & 46 & WCL & 3 & 0.82 \\
\hline & 47 & WCE & 1 & 0.69 \\
\hline & 48 & WNE & 44 & 0.67 \\
\hline & 49 & WCL & 13 & 0.49 \\
\hline & 50 & WNL & 8 & 0.69 \\
\hline & 51 & WNL & 1 & 0.23 \\
\hline & 52 & WNE & 18 & 0.29 \\
\hline & 53 & WCL & 2 & 0.23 \\
\hline & 54 & WCL & 6 & 0.56 \\
\hline & 55 & WNE & 20 & 0.24 \\
\hline
\end{tabular}


G. Leloudas et al.: WR star locations in their host galaxies

Table A.1. continued.

\begin{tabular}{|c|c|c|c|c|}
\hline & $\overline{\mathrm{ID}}$ & Subtype & Number & $\overline{F F}$ \\
\hline \multirow[t]{61}{*}{ M 83} & 56 & WNE & 15 & 0.69 \\
\hline & 57 & WNL & 9 & 0.73 \\
\hline & 58 & WCL & 5 & 0.42 \\
\hline & 59 & WNL & 14 & 0.83 \\
\hline & 60 & WNE & 6 & 0.75 \\
\hline & 61 & WCL & 2 & 0.84 \\
\hline & 62 & WCL & 4 & 0.44 \\
\hline & 63 & WCL & 5 & 0.28 \\
\hline & 64 & WNL & 10 & 0.31 \\
\hline & 65 & WCL & 4 & 0.52 \\
\hline & 66 & WNL & 8 & 0.59 \\
\hline & 66 & WCL & 4 & 0.59 \\
\hline & 67 & WCL & 5 & 0.57 \\
\hline & 68 & WCL & 3 & 0.44 \\
\hline & 69 & WCL & 2 & 0.28 \\
\hline & 70 & WCL & 2 & 0.25 \\
\hline & 71 & WCL & 3 & 0.62 \\
\hline & 72 & WCL & 1 & 0.75 \\
\hline & 73 & WNL & 2 & 0.74 \\
\hline & 74 & WNL & 52 & 0.94 \\
\hline & 74 & WCL & 179 & 0.94 \\
\hline & 75 & WCL & 1 & 0.39 \\
\hline & 76 & WNL & 1 & 0.66 \\
\hline & 77 & WCL & 2 & 0.61 \\
\hline & 78 & WNL & 11 & 0.91 \\
\hline & 79 & WCL & 5 & 0.66 \\
\hline & 80 & WNL & 1 & 0.33 \\
\hline & 81 & WCL & 3 & 0.46 \\
\hline & 82 & WCL & 4 & 0.61 \\
\hline & 83 & WCL & 2 & 0.56 \\
\hline & 84 & WCL & 9 & 0.92 \\
\hline & 85 & WCL & 4 & 0.58 \\
\hline & 86 & WNL & 9 & 0.96 \\
\hline & 86 & WCL & 24 & 0.96 \\
\hline & 87 & WCE & 3 & 0.41 \\
\hline & 88 & WCL & 1 & 0.39 \\
\hline & 89 & WCL & 3 & 0.79 \\
\hline & 90 & WCL & 2 & 0.82 \\
\hline & 91 & WCL & 3 & 0.45 \\
\hline & 92 & WCL & 3 & 0.31 \\
\hline & 93 & WNE & 10 & 0.19 \\
\hline & 94 & WNE & 23 & 0.64 \\
\hline & 95 & WNL & 1 & 0.43 \\
\hline & 96 & WNL & 2 & 0.79 \\
\hline & 97 & WNL & 5 & 0.90 \\
\hline & 98 & WCL & 4 & 0.38 \\
\hline & 99 & WNL & 1 & 0.71 \\
\hline & 100 & WNL & 5 & 0.89 \\
\hline & 101 & WNE & 11 & 0.45 \\
\hline & 102 & WNL & 9 & 0.84 \\
\hline & 103 & WNL & 29 & 0.80 \\
\hline & 104 & WCL & 3 & 0.73 \\
\hline & 105 & WNL & 8 & 0.89 \\
\hline & 106 & WCL & 6 & 0.85 \\
\hline & 107 & WNE & 15 & 0.78 \\
\hline & 108 & WNL & 2 & 0.58 \\
\hline & 109 & WCL & 7 & 0.83 \\
\hline & 110 & WCL & 3 & 0.64 \\
\hline & 111 & WCL & 3 & 0.32 \\
\hline & 112 & WCL & 2 & 0.78 \\
\hline & 113 & WCL & 5 & 0.38 \\
\hline
\end{tabular}


A\&A 518, A29 (2010)

Table A.1. continued.

\begin{tabular}{|c|c|c|c|c|}
\hline & ID & Subtype & Number & $F F$ \\
\hline \multirow[t]{61}{*}{ M 83} & 114 & WNE & 4 & 0.18 \\
\hline & 115 & WCL & 4 & 0.58 \\
\hline & 116 & WNL & 12 & 0.67 \\
\hline & 117 & WNE & 1 & 0.33 \\
\hline & 118 & WCL & 6 & 0.66 \\
\hline & 119 & WCL & 2 & 0.51 \\
\hline & 120 & WNE & 6 & 0.53 \\
\hline & 121 & WNE & 17 & 0.37 \\
\hline & 122 & WCE & 3 & 0.27 \\
\hline & 123 & WCL & 2 & 0.42 \\
\hline & 124 & WCL & 3 & 0.31 \\
\hline & 125 & WCL & 3 & 0.31 \\
\hline & 126 & WCE & 1 & 0.49 \\
\hline & 127 & WCE & 3 & 0.30 \\
\hline & 128 & WCL & 2 & 0.39 \\
\hline & 129 & WCE & 4 & 0.35 \\
\hline & 130 & WNE & 1 & 0.04 \\
\hline & 131 & WCL & 1 & 0.27 \\
\hline & 132 & WNE & 1 & 0.02 \\
\hline & 133 & cand. & $\ldots$ & 0.25 \\
\hline & 134 & cand. & $\ldots$ & 0.54 \\
\hline & 135 & cand. & $\ldots$ & 0.38 \\
\hline & 136 & cand. & $\ldots$ & 0.41 \\
\hline & 137 & cand. & $\ldots$ & 0.37 \\
\hline & 138 & cand. & $\ldots$ & 0.43 \\
\hline & 139 & cand. & $\ldots$ & 0.60 \\
\hline & 140 & cand. & $\ldots$ & 0.62 \\
\hline & 141 & cand. & $\ldots$ & 0.18 \\
\hline & 142 & cand. & $\ldots$ & 0.31 \\
\hline & 143 & cand. & $\ldots$ & 0.27 \\
\hline & 144 & cand. & $\ldots$ & 0.82 \\
\hline & 145 & cand. & $\ldots$ & 0.73 \\
\hline & 146 & cand. & $\ldots$ & 0.75 \\
\hline & 147 & cand. & $\ldots$ & 0.51 \\
\hline & 148 & cand. & $\ldots$ & 0.95 \\
\hline & 149 & cand. & $\ldots$ & 0.91 \\
\hline & 150 & cand. & $\ldots$ & 0.95 \\
\hline & 151 & cand. & $\ldots$ & 0.95 \\
\hline & 152 & cand. & $\ldots$ & 0.87 \\
\hline & 153 & cand. & $\ldots$ & 0.86 \\
\hline & 154 & cand. & $\ldots$ & 0.79 \\
\hline & 155 & cand. & $\ldots$ & 0.31 \\
\hline & 156 & cand. & $\ldots$ & 0.89 \\
\hline & 157 & cand. & $\ldots$ & 0.76 \\
\hline & 158 & cand. & $\ldots$ & 0.42 \\
\hline & 159 & cand. & $\ldots$ & 0.91 \\
\hline & 160 & cand. & $\ldots$ & 0.96 \\
\hline & 161 & cand. & $\ldots$ & 0.30 \\
\hline & 162 & cand. & $\ldots$ & 0.53 \\
\hline & 163 & cand. & $\ldots$ & 0.82 \\
\hline & 164 & cand. & $\ldots$ & 0.25 \\
\hline & 165 & cand. & $\ldots$ & 0.59 \\
\hline & 166 & cand. & $\ldots$ & 0.28 \\
\hline & 167 & cand. & $\ldots$ & 0.16 \\
\hline & 168 & cand. & $\ldots$ & 0.86 \\
\hline & 169 & cand. & $\ldots$ & 0.33 \\
\hline & 170 & cand. & $\ldots$ & 0.87 \\
\hline & 171 & cand. & $\ldots$ & 0.82 \\
\hline & 172 & cand. & $\ldots$ & 0.35 \\
\hline & 173 & cand. & $\ldots$ & 0.81 \\
\hline & 174 & cand. & $\ldots$ & 0.37 \\
\hline
\end{tabular}


G. Leloudas et al.: WR star locations in their host galaxies

Table A.1. continued.

\begin{tabular}{|c|c|c|c|c|}
\hline & ID & Subtype & Number & $F F$ \\
\hline \multirow[t]{47}{*}{ M 83} & 175 & cand. & $\ldots$ & 0.83 \\
\hline & 176 & cand. & $\ldots$ & 0.58 \\
\hline & 177 & cand. & $\ldots$ & 0.74 \\
\hline & 178 & cand. & $\ldots$ & 0.74 \\
\hline & 179 & cand. & $\ldots$ & 0.99 \\
\hline & 180 & cand. & $\ldots$ & 0.43 \\
\hline & 181 & cand. & $\ldots$ & 0.15 \\
\hline & 182 & cand. & $\ldots$ & 0.42 \\
\hline & 183 & cand. & $\ldots$ & 0.63 \\
\hline & 184 & cand. & $\ldots$ & 0.63 \\
\hline & 185 & cand. & $\ldots$ & 0.91 \\
\hline & 186 & cand. & $\ldots$ & 0.62 \\
\hline & 187 & cand. & $\ldots$ & 0.60 \\
\hline & 188 & cand. & $\ldots$ & 0.37 \\
\hline & 189 & cand. & $\ldots$ & 0.93 \\
\hline & 190 & cand. & $\ldots$ & 0.92 \\
\hline & 191 & cand. & $\ldots$ & 0.77 \\
\hline & 192 & cand. & $\ldots$ & 0.67 \\
\hline & 193 & cand. & $\ldots$ & 0.34 \\
\hline & 194 & cand. & $\ldots$ & 0.91 \\
\hline & 195 & cand. & $\ldots$ & 0.96 \\
\hline & 196 & cand. & $\ldots$ & 0.88 \\
\hline & 197 & cand. & $\ldots$ & 0.67 \\
\hline & 198 & cand. & $\ldots$ & 0.85 \\
\hline & 199 & cand. & $\ldots$ & 0.67 \\
\hline & 200 & cand. & $\ldots$ & 0.84 \\
\hline & 201 & cand. & $\ldots$ & 0.55 \\
\hline & 202 & cand. & $\ldots$ & 0.33 \\
\hline & 203 & cand. & $\ldots$ & 0.64 \\
\hline & 204 & cand. & $\ldots$ & 0.67 \\
\hline & 205 & cand. & $\ldots$ & 0.84 \\
\hline & 206 & cand. & $\ldots$ & 0.89 \\
\hline & 207 & cand. & $\ldots$ & 0.88 \\
\hline & 208 & cand. & $\ldots$ & 0.87 \\
\hline & 209 & cand. & $\ldots$ & 0.79 \\
\hline & 210 & cand. & $\ldots$ & 0.44 \\
\hline & 211 & cand. & $\ldots$ & 0.66 \\
\hline & 212 & cand. & $\ldots$ & 0.88 \\
\hline & 213 & cand. & $\ldots$ & 0.54 \\
\hline & 214 & cand. & $\ldots$ & 0.82 \\
\hline & 215 & cand. & $\ldots$ & 0.70 \\
\hline & 216 & cand. & $\ldots$ & 0.47 \\
\hline & 217 & cand. & $\ldots$ & 0.22 \\
\hline & 218 & cand. & $\ldots$ & 0.51 \\
\hline & 219 & cand. & $\ldots$ & 0.42 \\
\hline & 220 & cand. & $\ldots$ & 0.39 \\
\hline & 221 & cand. & $\ldots$ & 0.01 \\
\hline \multirow[t]{14}{*}{ NGC 1313} & 1 & WNE & 1 & 0.37 \\
\hline & 2 & WCE & 1 & 0.30 \\
\hline & 3 & WNL & 1 & 0.06 \\
\hline & 7 & WNL & 1 & 0.03 \\
\hline & 8 & WNL & 1 & 0.16 \\
\hline & 9 & WNE & 1 & 0.28 \\
\hline & 10 & WCE & 2 & 0.30 \\
\hline & 11 & $\mathrm{WNE}^{a}$ & 1 & 0.00 \\
\hline & 11 & WCE & 1 & 0.00 \\
\hline & 12 & WCE & 1 & 0.47 \\
\hline & 13 & WNL & 1 & 0.11 \\
\hline & 14 & $\mathrm{WNE}^{a}$ & 1 & 0.67 \\
\hline & 15 & WCE & 1 & 0.34 \\
\hline & 16 & WCE & 1 & 0.71 \\
\hline
\end{tabular}


A\&A 518, A29 (2010)

Table A.1. continued.

\begin{tabular}{|c|c|c|c|c|}
\hline & $\overline{\mathrm{ID}}$ & Subtype & Number & $\overline{F F}$ \\
\hline \multirow[t]{61}{*}{ NGC 1313} & 17 & WNE & 1 & 0.71 \\
\hline & 18 & WNL & 1 & 0.22 \\
\hline & 19 & WNE & 1 & 0.02 \\
\hline & 20 & WCE & 1 & 0.70 \\
\hline & 21 & WNL & 1 & 0.74 \\
\hline & 22 & WNL & 1 & 0.74 \\
\hline & 23 & WNE & 1 & 0.46 \\
\hline & 24 & WNL & 1 & 0.62 \\
\hline & 25 & WNL & 1 & 0.46 \\
\hline & 28 & cand. & $\ldots$ & 0.38 \\
\hline & 29 & WNE & 1 & 0.39 \\
\hline & 30 & WNL & 1 & 0.03 \\
\hline & 31 & WCE & 1 & 0.78 \\
\hline & 32 & WNL & 1 & 0.03 \\
\hline & 33 & WNE & 3 & 0.86 \\
\hline & 34 & cand. & $\ldots$ & 0.91 \\
\hline & 35 & WCE & 1 & 0.89 \\
\hline & 37 & WCE & 1 & 0.99 \\
\hline & 39 & WNL & 1 & 0.52 \\
\hline & 40 & WNE & 1 & 0.84 \\
\hline & 41 & WCE & 1 & 0.71 \\
\hline & 42 & WCE & 1 & 0.68 \\
\hline & 44 & WNE & 1 & 0.53 \\
\hline & 45 & WNE & 1 & 0.41 \\
\hline & 46 & WNE & 1 & 0.62 \\
\hline & 47 & WNL & 1 & 0.62 \\
\hline & 48 & WCE & 2 & 0.58 \\
\hline & 49 & WCE & 1 & 0.58 \\
\hline & 50 & WCE & 1 & 0.89 \\
\hline & 51 & WNE & 1 & 0.83 \\
\hline & 52 & WNE & 1 & 0.60 \\
\hline & 53 & WNE & 1 & 0.80 \\
\hline & 54 & cand. & $\ldots$ & 0.60 \\
\hline & 55 & WNE & 1 & 0.51 \\
\hline & 56 & WCE & 1 & 0.89 \\
\hline & 57 & WCE & 1 & 0.91 \\
\hline & 58 & $\mathrm{WNE}^{a}$ & 3 & 0.97 \\
\hline & 59 & $\mathrm{WNE}^{a}$ & 1 & 0.95 \\
\hline & 59 & WCE & 1 & 0.95 \\
\hline & 60 & WCE & 1 & 0.97 \\
\hline & 61 & WCE & 3 & 0.18 \\
\hline & 62 & WCE & 1 & 0.18 \\
\hline & 63 & cand. & $\ldots$ & 0.56 \\
\hline & 64 & WCE & 3 & 0.95 \\
\hline & 65 & cand. & $\ldots$ & 0.98 \\
\hline & 66 & WCE & 2 & 0.96 \\
\hline & 67 & $\mathrm{WNE}^{a}$ & 1 & 0.96 \\
\hline & 67 & WCE & 1 & 0.96 \\
\hline & 68 & cand. & $\ldots$ & 0.45 \\
\hline & 70 & WNE & 1 & 0.28 \\
\hline & 71 & WNE & 1 & 0.47 \\
\hline & 72 & WNL & 1 & 0.40 \\
\hline & 73 & WNL & 1 & 0.44 \\
\hline & 75 & cand. & $\ldots$ & 0.00 \\
\hline & 76 & WNE & 1 & 0.22 \\
\hline & 77 & WNL & 1 & 0.36 \\
\hline & 78 & WNE & 1 & 0.30 \\
\hline & 79 & WNE & 1 & 0.04 \\
\hline & 80 & cand. & $\ldots$ & 0.54 \\
\hline & 81 & WCE & 1 & 0.52 \\
\hline & 82 & cand. & $\ldots$ & 0.64 \\
\hline
\end{tabular}

Page 14 of 15 
G. Leloudas et al.: WR star locations in their host galaxies

Table A.1. continued.

\begin{tabular}{rrrrr}
\hline \hline & ID & Subtype & Number & $F F$ \\
\hline NGC 1313 & 83 & WNL & 1 & 0.53 \\
& 85 & WNL & 1 & 0.53 \\
& 86 & cand. & $\ldots$ & 0.15 \\
& 87 & cand. & $\ldots$ & 0.20 \\
& 88 & WCE & 2 & 0.00 \\
& 90 & WNL & 1 & 0.33 \\
& 91 & WNL & 1 & 0.17 \\
& 92 & WNL & 1 & 0.32 \\
& 94 & WNL & 1 & 0.09 \\
\hline
\end{tabular}

Notes. ${ }^{(a)}$ These are stars classified as WN5-6 and we have included them in the WNE distribution. 